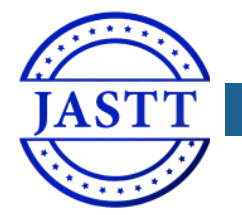

\title{
Internet of Robotic Things: A Review
}

\author{
Sibar Jameel Khalid \\ Department of Computer Science - Faculty of Science, University of Zakho, Kurdistan Region of Iraq, \\ sibar.khalid@stud.uoz.edu.krd
}

\begin{abstract}
The Internet of Things (IoT) gives a strong structure for connecting things to the internet to facilitate Machine to Machine (M2M) communication and data transmission through basic network protocols such as TCP/IP. IoT is growing at a fast pace, and billions of devices are now associated, with the amount expected to reach trillions in the coming years. Many fields, including the army, farming, manufacturing, healthcare, robotics, and biotechnology, are adopting IoT for advanced solutions as technology advances. This paper offers a detailed view of the current IoT paradigm, specifically proposed for robots, namely the Internet of Robotic Things (IoRT). IoRT is a collection of various developments such as Cloud Computing, Artificial Intelligence (AI), Machine Learning, and the (IoT). This paper also goes over architecture, which would be essential in the design of Multi-Role Robotic Systems for IoRT. Furthermore, includes systems underlying IoRT, as well as IoRT implementations. The paper provides the foundation for researchers to imagine the idea of IoRT and to look beyond the frame while designing and implementing IoRT-based robotic systems in real-world implementations.
\end{abstract}

Keywords: Internet of Robotic Things (IoRT), Robotics, Sensors, Cloud Computing, Artificial Intelligence (AI), Machine Learning $(M L)$.

Received: May 24 $4^{\text {th }}, 2021 /$ Accepted: July 28 ${ }^{\text {th }}$, / Online: August $6^{\text {th }}, 2021$

\section{INTRODUCTION (HEADING 1)}

So far, the IoT and robotics culture has been supported by different but highly complementary goals; the first aimed to promote information services for detection, tracking, and ubiquitous surveillance, and the second to create movement, interaction, and interaction behavior. [1] As a result, it is increasingly believed that the development of an (IoRT) that combines the results of the two cultures will have great added value.

Early signs of IoRT integration can be found in control concepts of clustered and heterogeneous robots such as networked robotics or robotic ecology, as well as strategies such as ubiquitous robotics and cloud robotics hosts resourceintensive technologies on the server-side [2][3][4] [5] [6] [7]. In an Allied Business Intelligence (ABI) research report, the term "Robotic Internet of Things" was coined to describe the structure in which sensor data from various sources is combined, interpreted using local and distributed information, and used to monitor and verify things in the physical world [8].

This cyber-physical IoT perspective has led to better business performance by leveraging IoT sensors and data analysis tools to enable robots to better understand the situation. Intelligent transportation and robotic assistants are two use cases [9]. The previous use of the word IoRT in research corresponded to alternative approaches, such as the "robotic view of the Internet of Things" aimed at stable team communication, and robots are simply additional sensors [8] [10] [11] [12] [13].

Fig. 1 shows two non-robot assists in the development of distributed robotic systems: cloud computing and the Internet of Things. Three ideas underly IoT technology: (1) sensors embedded in the environment and on our body parts; (2) connected smart objects communicating through machine-tomachine (M2M); and (3) data analytics and semantic technologies that transform raw sensor data. Cloud computing provides on-demand network access to a pool of virtualized hardware resources (computers, storage) or higher-end appliances. The cloud was utilized by the IoT group to provide customizable IoT network resources that control sensor data access (raw, archived, or aggregated). Managing billions of IoT device data streams across several clustered data centers creates concerns about latency, reaction time, large input bandwidth needs, and data privacy [14]-[16]. Edge computing (also known as fog computing or cloud applications) brings distributed 
computing power on demand closer to the edge of the network, to data generators. [17]. The cloud framework has also been adopted by the robot community called cloud robotics to offload resource-intensive tasks, through data and information exchange between robots app store models [18] [7] [19] [20]. While cloud robotics and IoRT overlap, the former focuses more on providing computing power and accessible storage networks for data and information, while the latter focuses more on connectivity, M2M, and intelligent data processing [21] [8].

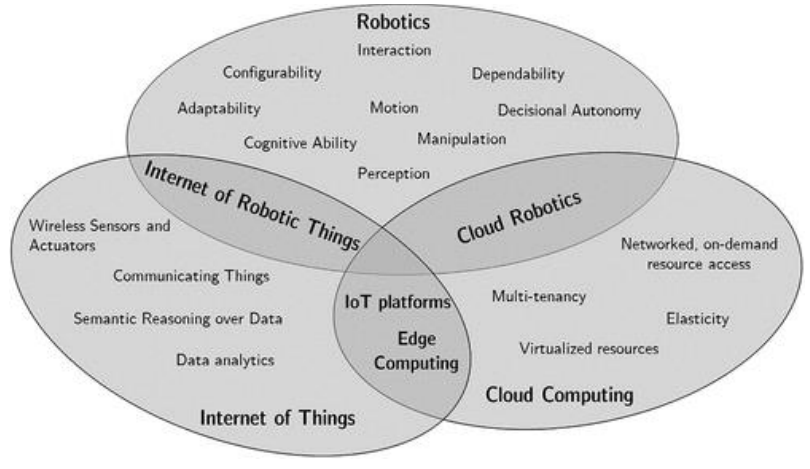

Fig. 1. Three concepts of IoT technologies [22].

The purpose of this article is to provide a quick overview of the idea of a robotic IoT, including its general design, advantages, and challenges, as well as some realistic use cases and guidance, in the hopes of raising awareness and encouraging future research. The following is how the rest of the article is organized: chapter 2 is a summary of recent work. Chapter 3 introduces the reader to the idea behind the robotic Internet of Things, concepts, capabilities, and IoRT architecture. Chapter 4 highlights the five key technologies enabling IoRT, while Chapter 5 discusses unresolved issues and challenges. Chapter 6 describes some important practical applications of IoRT. Discussion and Conclusion summarized in Sections $7 \& 8$.

\section{RELATED WORK}

The rapid proliferation of IoT-based applications has recently resulted in the definition of IoT trends with a particular focus on the industrial situation [23] [24] [25] [26] [27]. In each of these studies, the author's abstracted patterns from current IoT goods and innovations, and in some cases, split model approaches and best - practices into two groups, respectively patterns and pattern candidates (not fully formulated templates that can be promoted to patterns in the future). Moreover, in robotics, typical issues are often solved by modularity, which can be viewed as the hardware equivalent of the software pattern [28]. Even so, in the sense of system-level hardware architecture, the issue of defining design and manufacturing patterns was directly discussed, with the authors attempting to rewrite the initial concept of software design patterns for the hardware situation [29]. A parallel strategy was used for cloud infrastructure and for what are known as smart contracts in financial services [30] [31].

In the comprehensive work of [23] [32] [33], the authors reviewed major works on pattern recognition, description, and implementation to select a collection of elements, some necessary and others optional, useful to define IoT patterns. There are, in general, the name and aliases to identify the pattern, the symbol to connect a graphical component with the pattern in a diagram, a problem section to describe the issue with which the pattern is built, the context to explain the situation wherein the problem exists and any constraints, and the forces represented by potentially conflicting considerations to keep in mind when selecting a pattern [34] [35].

\section{INTERNET OF ROBOTIC THINGS}

IoRT is about the convergence of Smart Field capabilities and autonomous agents in the technical IoT system (robot). Fig. 2 clearly illustrates this principle. We refer to smart zones as applications such as smart rooms, smart factories, smart buildings, and smart cities. The monitoring of states and processes in a specified control environment is the key aspect of these applications. Most functions involve maintaining necessary environmental factors such as room temperature and humidity, often using complex heating, ventilation, and air conditioning (HVAC) systems, or using control conditions (such as turning on the air) with simple sensors and actuators. air conditioning or opening windows with simple controls and turning the heating on and off at the right time). When regular electricity rates or power peaks are low, one of the aims is to turn off the power, for example, or the washing machine. This implies that energy use is tracked by looking for people who are carrying household appliances [36].

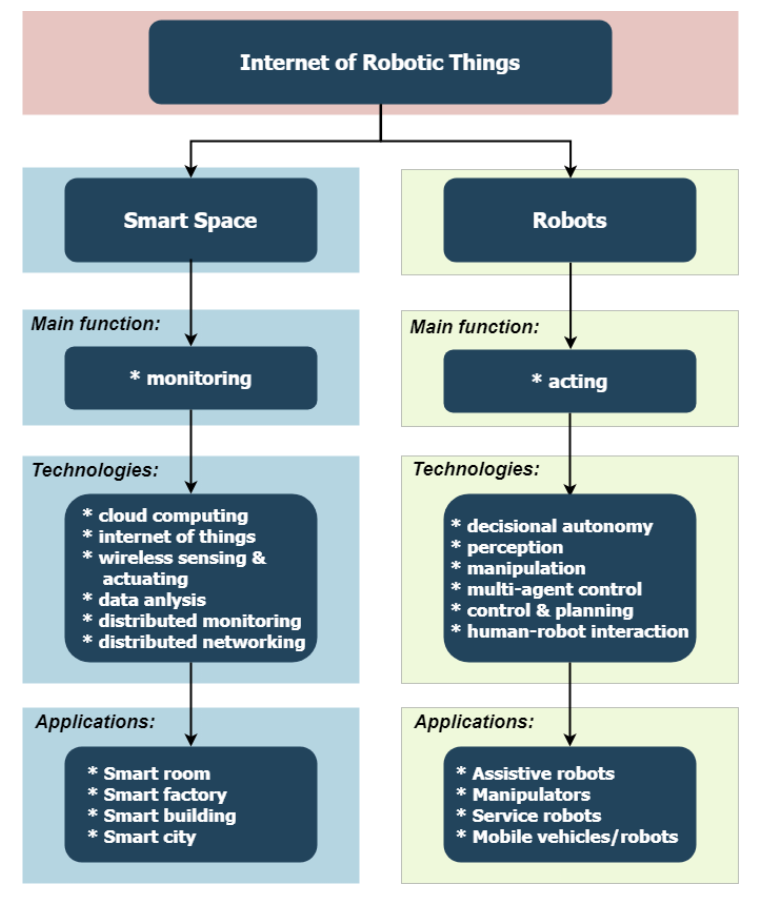

Fig. 2. The block-scheme of the IoRT[37]

The Smart Space lacks agents that can undertake activities in the room despite the availability of fundamental monitoring functions and executive processes (moving objects, performing certain operations or services, etc.). Examples of such agents are assistant robots, manipulators, utility robots, and personal cars/robots. By integrating and developing the capabilities of the 
Smart Space and robots, the development of autonomous agents in the Smart Space contributes to the definition of IoRT. Modern robotics usually focuses only on increasing robot autonomy, improving vision criteria with robotic sensors, and on-board data processing that allows robots to perform tasks autonomously. The atmosphere in the room where the robots perform certain activities, various sensors (RFID, presence detectors, surveillance cameras, magnetic sensors, IR/audio signals, etc.) and computer resources (smartphones, routers, etc.). Therefore, all robots and intelligent space, whose capabilities and functions are enhanced by the capabilities of the intelligent environment, benefit from such a combination when agents (robots) often perform complex operations, in addition, to control functions in intelligent space perform basic operations with simple mechanisms. Robots can receive instructions from a smart space (such as a smart building or smart factory), which monitors progress and gives the robot instructions from a network of smart environmental sensors, such as optimal navigation, avoiding obstacles/collisions, or successful interaction between humans and robots, thanks in part to this combination As a result, the robotic Internet of Things is a much more advanced version of the IoT, and it also enables the integration of new technologies such as cloud computing, sensors, and wireless control, data analytics, distributed control and communication of the Smart Space such as decision control, detection, manipulation, multi-agent control, control, and programming as well as human-robot interaction - from Smart Field (Fig. 2) [37].

\section{A. Concepts}

Artificial intelligence (AI), robotics, machine learning, and swarm technology will power the next stage of IoT application growth. Robotics systems have historically given a programmable dimension to computers programmed to perform laborious and routine tasks. Artificial intelligence and machine learning enable these computers to work by making decisions and learning algorithms rather than programming.

Industrial IoT is a branch of the Internet of Things in which edge nodes, computing units, and networks communicate with their surroundings to produce data that can be used to optimize processes. IoRT technologies can be realistically allocated in this environment by autonomous functions and IoT [38]. A wired robot is a robotic computer that is linked to a networking network, such as the Internet or a local area network (LAN). The network could be wired or wireless, and it could use any of several protocols, including TCP, UDP, or 802.11. Many new technologies, ranging from automation to discovery, are currently being developed. The sensor network broadens the robots' efficient sensing range. This allows them to communicate with each other over long distances to organize their activities. In turn, robots can install, repair and maintain a network of sensors, increasing its reliability and availability [39] [40].

The development of a science foundation that connects connectivity for managing and activating new capabilities is a popular problem in the two subclasses of networked robots. A robot is usually a closed system(s) with large capacities. Wireless networks are needed for networked robots to share data among multiple robots [41]. This communication has significant consequences for task-sharing among robots, such as enabling teleoperation. These systems' evolution has now entered the user industry, for example, to enable online meetings and telepresence healthcare equipment [42]. Cloud robotic systems have also arisen to address the shortcomings of networked robotics by using scalable capabilities provided by cloud computing [43].

IoRT goes beyond networked and collaborative/cloud robots. Integrate different smart devices into a distributed architecture with systems running both in the cloud and at the edge. IoRT examines the different ways in which IoT technologies and robotic "devices" are combined today to provide advanced robotic capabilities [38].

The fast expansion of different radio access methods to link smart devices to the edge has resulted in the rise of heterogeneous mobile networks that require extensive configuration, administration, and maintenance. AI approaches enable the near-perfect integration of IoRT processing systems into IoT applications.

The combination of advanced discovery/action, networking, local and distributed computing takes the original vision of IoT to a whole new dimension. The definition enables the following baseline attributes to be summarized. Create and specify the properties of robotic technologies that set them out as a unique class of IoT services. Show how the IoT model improves the core features of robotics technology, such as acceleration, stability, manipulation, intellect, and autonomy [44]. Show how IoT and robotics technology works together to include ambient sensing, knowledge, and localization. Integration of IoT, semantic processing, and artificial intelligence technology is part of recent trends [38].

\section{B. IORT Abilities}

The multidiscipline design of the IoRT fits the same pattern as the IoT in that it has sophisticated robotic technologies, resulting in the emergence of interdisciplinary applications for multiple and diverse disciplines.

Technically, IoRT relies on the basic robot capabilities, which are broadly categorized into three: basic abilities, higherlevel abilities, and system-level abilities [8].

\section{- Basic Abilities:}

- Perception Ability: In terms of space, time, and kind of information, IoT sensor and data analytics technologies offer robots a broader horizon than local, onboard sensing. Putting sensors on mobile robots, on the other hand, helps them to be positioned in a scalable and interactive manner, enabling sophisticated active sensing strategies [8].

- Motion Ability: One of the primary benefits of robotic systems is their ability to travel. While mechanical architecture is the most important factor in determining the inherent efficiency of robot mobility, IoT networking may assist mobile robots in controlling automated doors and elevators, for example, in logistics robotics and 
support applications [45]. IoT software utilities can assist in the management of distributed robotic systems in large-scale applications including M2M and network protocols, last-mile deployment, precision farming, and environmental monitoring [46].

○ Manipulation Ability: While IoT's major objective is to sense the world, robots' major objective is to change it. Using their end effectors, robots will grab, raise, catch, and shift objects. The sequence of the pairs applied to the joints may be calculated using inverse kinematics when the robot learns the important aspects of the item, such as its direction and shape [8].

\section{- Higher Level Abilities:}

- Decisional Autonomy: Decision autonomy refers to the system's ability to determine the best course of action to complete its tasks. This is frequently neglected in IoT middleware: apps use an execution API called smarts, which hides the intrinsic complexity [8].

- Interaction Ability: Interaction ability refers to a robot's ability to communicate mechanically, cognitively, and socially with controllers or other systems in a production environment. The Interaction ability reflects on how IoT technology can improve humanrobot interaction in the industrial world. IoT devices can improve the robustness of humanrobot interaction in the manufacturing world. The (IoT) will include details on the location and condition of parts and facilities to help decipher natural language commands, which are often ambiguous or contain tacit assumptions [47][48].

- Cognitive Ability: Given the exponential increase in robot participation in many areas of life, understanding how robots manipulate people's emotions during human-robot encounters is key to driving robot adoption in society. Mental health, in especially, is considered to be the area where robot technology can have the greatest impact in a short time. Cognitive robots can grasp the interaction between themselves and the world, between objects, and the potential effect of their behavior through reasoning about and inferring information from experience. Inside a smart warehouse, incorporating edge computing facilitates the expansion of the cloud platform's computing power, network bandwidth, and storage to the IoT edge, as well as upstream and downstream delivery of data in resource planning and production and development processes [49]. Workplace risk factors (for example, uncomfortable positions, unnecessary activity, and repeated actions) are rising issues in the industrial sector. Throughout this regard, interactive robotics designed specifically for manufacturing activities has emerged as an appealing solution to the issue. The biggest advantage of IoT in terms of cognitive capacity is the infrastructure and technology of AI, as well as the recognition of emotions and the interaction of the Emotional Interaction Intelligence Robot (iRobot) with the cloudbased IoT network. performances are both the assembly of the chip and the productivity of development [49][48].

\section{- System-Level Abilities}

- Configurability: Configurability is described as the ability of a robotic system to be customized for specific tasks or reconfigured for various tasks. IoT is useful in the manufacturing context for software configurability and interactive configuration of several computers that contribute different functionality and collaborate to execute complex tasks or jobs. For example, to save changeover and set-up time, an assembly line has been built as a multi-agent system with personality capabilities [50]. The (IoT) and artificial intelligence (AI) become engines of technological progress in smart manufacturing, contributing to economic well-being and improving people's lives. In intelligent storage, edge computing consolidation extends the power of cloud computing, networking, and storage speeds to the edge of the IoT, as well as streamlining resource planning and up and down data delivery during the production cycle and development. In addition, cloud-based Internet of Things (IoT) architecture and its main competitor, artificial intelligence technology, enable Intelligent Affective Interaction Intelligent Robot (iRobot) to solve the psychological problems of the user [48].

- Adaptability: Adaptability refers to a system's ability to respond to a variety of job situations, conditions, surroundings, and needs. This requires the ability to react to unanticipated circumstances, flaws, evolving roles and conditions, and unpredictable human behavior. Perception, decision-making, and configuration skills are primary enablers of adaptability [8].

- Dependability: The dependability of the hardware and software modules of the robots, the assurances of protection while interacting with people, and the extent to which devices will execute their work in the event of an 
accident or other unanticipated circumstances are all aspects of reliability. One method of ensuring dependability is forecasting faults or conflicts. In a factory, for example, if the operator goes too close to the robot, it will stop. IoT technologies can help by adding practical tools. Rampa et al. [51] exploited radio field interference to forecast a user's position using a modest array of transceivers in a robotic cell. Sensors have been placed into the clothes and helmets of other researchers. By integrating data from fixed cameras and onboard sensors with historical data of human trajectories Qian et al. [52] developed a probabilistic paradigm to prevent conflicts between human and robotic motions [8].

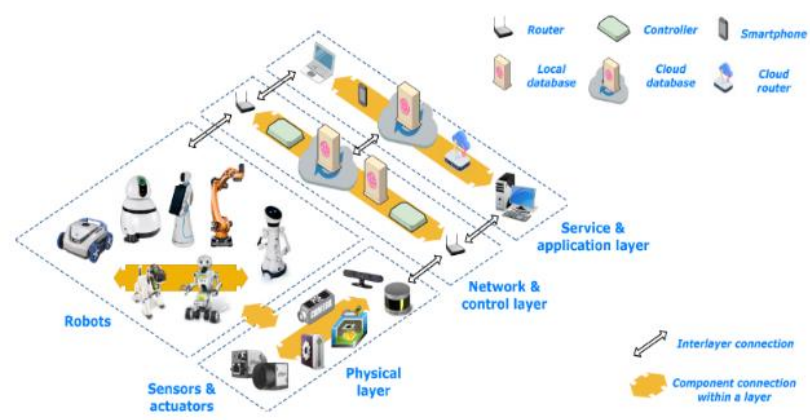

Fig. 3. The architecture for the IoRT framework [37].

\section{The Architecture of IORT}

In this part, we provide a three-tier IoRT reference architecture. Fig. 3 shows the overall architecture of the device, which consists of three main layers: the first physical, the second network and management, and the third services and applications.

- Physical Layer: The physical layer consists of different robots, sensors, and actuators. Robots are autonomous entities that can interact with one another and form a multi-robot system to accomplish a shared purpose. Sensors in the Physical layer are sensors that track important environmental parameters. The primary way to connect robots and sensors is at the network and control level, where different components can interact and control processes in an intelligent environment using standard protocols [37].

- Network and Control Layer: The network and management layer may include numerous routers, switches, local and cloud storage (servers), and networking and management protocols. To preprocess and archive data from sensors, actuators, and robots, both local storage (for each smart space or smart building in general) and distant storage (in the clouds) can be employed [37].
- Service and Applications Layer: The installation and execution of joint and customized programs for sensing, processing, and controlling all parameters and environmental agents (sensors, actuators, and robots) in smart space were carried out at the service and application level by the interconnected IoRT. In addition to this level of modern sensory processing algorithms, artificial intelligence (AI) and machine learning (ML) algorithms can be used to increase IoRT efficiency by transferring low-latency data to modern database devices [37].

\section{IORT TECHNOLOGIES}

This section presents various technologies, such as IoRT, that enable the design and execution of sophisticated robots. IoRT has many technologies such as sensors and actuators, communication technologies, processing, and sensors/ actuators data fusion, environments, objects, things, modeling and dynamic mapping, virtual and augmented reality, voice recognition, voice control, orchestration, decentralized cloud, adaptation, machine learning, end to an end operation and internet technologies safety and security framework, blockchain [38], [53], [54].

Below are brief descriptions of some of the IoRT technologies that allow building, installation, and deploy IoT applications.

\section{A. Actuators and Sensors}

Sensors and actuators have valuable features both within and outside of the IoRT construction components. Robotic Interaction Services (RoIS) also describes the use of the brick view. RoIS summarizes the features of the robot-human-robot (HRI) interface and charts in the service robot. Human detection and identification (HRI) components are conceptual functional aspects performed by physical equipment such as sensors installed on a robot and/or surroundings. IoRT applications are subjected to sunshine, darkness, rain, fog, dust, and other environmental conditions, which requires the use of low-cost solid-state semiconductor (CMOS) image sensors that are stable under a variety of situations, such as [55].

For scanning the road surface (horizontal projection) and object identification, the sensors must have a high resolution and sensitivity (vertical projection). RoIS feature enables the development of software that can be deployed on both gateways and platforms but is particularly applicable to HRI situations. It is intended to allow the production and implementation of IoRT systems on a variety of platforms, such as smartphones and smartwatches, smart meters, and smart cars. Future IoRT functions would necessitate more height detail, 3D visualization, and sensor/actuator fusion[56].

LIDAR systems are ineffective for close-in monitoring, so autonomous robotic things must be fitted with radars. When the environment is clouded by smoke, dust, or other atmospheric circumstances, a radar scanner is an exciting tool for collision avoidance. A 3D representation of an environment based on or customized to existing/new sensor technologies is required for robotic equipment [57] [38]. 


\section{B. Technologies of Communication}

IoRT's connectivity architecture requires new techniques that allow shared real-time computing and data stream sharing. The Narrowband IoT (NB-IoT) low power small areas infrastructure for IoT implementations is based on $3 \mathrm{GPP}$ standards and uses the same 4G/LTE network.

Bluetooth is a low-power, low-cost wireless networking technology that is built on the IEEE 802.15.1 specification. LoRaWAN is a network protocol designed for end-nodes that are operated by batteries. The attached node's battery life is usually very long, lasting up to ten years All connections are bidirectional, multicast compatible, and in star-to-star topology. Ten-year battery life and low-cost devices will enable a wide range of applications that can support many low-efficiency devices [38].

\section{Voice Recognition, Voice Control}

Speech recognition and control systems play an important part in facilitating improved human-robot interactions. With the development of IoRT, there seems to be a need for collaboration between robots, so a suitable platform is needed for humanrobot collaboration to enhance digital experiences IoRT-based robotics is widely used in tourism planning and guidance, patient care, in factories, homes, search and rescue, schools, etc. It can be used in a variety of applications, including manufacturing processes. To do this, IoRT systems must be equipped with simple interfaces that allow communication between machines and humans. Speech recognition and response technologies are critical in this sense. The structures should be modular and versatile enough to remove noise by using data from the robot's movements and gestures. Microphone efficiency and speech recognition algorithms need to be greatly improved to suppress external noise IoRT systems can be equipped with multi-channel systems using advanced techniques such as sidelobe suppression and field noise suppression [58][22].

\section{Machine Learning}

In recent years, the IoT has begun to adopt machine learning and deep learning approaches, methodologies, and algorithms to add advanced knowledge to connected devices. Machine learning, a subfield of computer science and artificial intelligence, originated from model analysis and statistical learning. This allows computers to learn from data by experimenting with building models to simulate and explore datasets. In the coming years, machine learning may replace human learning for data processing and prediction [59].

Through IoT and machine learning, IoRT systems are becoming more efficient, allowing them to easily adjust to changing surroundings, execute high-level dynamic computations in real-time, and increase performance. At the core of the IoRT learning service is a key issue in the design and execution of learning models, which must be efficiently constructed to satisfy the needs of computing resources placed at network nodes and then adapted to the underlying fabric lowlevel data mining. ANNs were proposed by Razafimandimby et al [60] to enable worldwide communication across IoRT robotic systems. Neural networks can strike a balance between targeted network coverage and QoS connection [22].

\section{E. Frameworks for Security}

The biggest barrier to effective IoRT implementation in the real world is protection and security to enable efficient collaboration with networks, sensors, and other robots, as well as to control all kinds of illegal behavior and simulate different network attacks and intrusions [61], [62]. To transmit information end-to-end securely and reliably, IoRT networks need to be built. In terms of honesty and secrecy, security systems can relay information safely obtained by robotics. IoRT poses a significant threat to companies that collect data from robotic systems. As a result, multiple devices are needed to protect data from illegal access. Furthermore, IoRT networks are still wired to the Internet, exposing them to new types of data breach attacks [63], [64].

IoRT systems must provide a physical access control mechanism to validate data, ensure confidentiality and trust, and most importantly protect the confidentiality of information [22].

\section{Challenges OF IORT}

The integration of robotic technology with IoT systems and cloud computing is the goal of IoRT. The interaction between the study domains of IoT, cloud computing, and robotics is continually expanding as a result of this convergence [65].

Concerns about permission networks, maintaining access, infrastructure, and interacting with other robots, and following organized paths make it difficult for multiple robots to cooperate. HRI-defined human movements must be adapted by intelligent robots. In recent years, modern types of HRI such as gaze tracking, voice interaction, and biological recognition have been investigated, but these have yet to be tested as most of them have only been studied in research laboratories [66].

Advances in IoRT technology can hold the key to a modern, better way of managing industrial operations. Remote working has recently received a lot of coverage thanks to the many advantages it provides. Remote working helps increase productivity through a better work-life balance. Students are rewarded higher in a classroom environment for using social robots deployed in an IoRT scenario. Remote education is extremely useful for students who are unable to leave their homes due to sickness. The educational relationship between people and robots has to be further developed [67] [68] [69].

Energy demand is one of the most pressing problems in the industrial energy consumption period. The challenge in measuring and optimizing energy quality in smart environments stems primarily from a lack of precise comprehension of energy consumption behavior [70]. To address this concern, considerable focus and effort must be devoted to gathering data on energy usage from smart sensors. To achieve sustainable processes, this knowledge must be incorporated into supply management [71].

Significant volumes of data are processed in IoRT systems, which causes cyber-security issues. In such cases, the IoRT network can need additional calculation and storage capacities. Insecure contact between users and robots is one of the most serious cyber-security issues [72]. 
In the world of manufacturing, the collaboration between humans and robots is a very important issue. Production data must be saved in the same format and material provided at the time of creation. Data must be shared using the same encrypted protocol with any other compatible industrial infrastructure anywhere in the world. Output and other industrial management applications should be kept secret and closely supervised. The information history can also hold the key to ensuring cyberphysical protection [73].

Addressing the challenges of cyber-physical security is imperative to advance the evolution of IoRT networks into smart spaces. Security issues related to CPS and robot connectivity require further investigation. Connecting smart devices is key to further progress in both manufacturing and science [74].

\section{IORT APPLICATIONS}

Some businesses are profiting from the fourth industrial revolution's exponential development, which combines automation, CPS, and the cloud. IoRT systems have many benefits over conventional robotic implementations in this sense. Examples: transferring resource-intensive activities to the cloud, accessing large amounts of data, and exchanging information with other robots.

The sections that follow detail the many areas where IoRT solutions are addressed and introduced. Table 1 , in the following sections, details the situations discussed in this section, showing the different types of advanced technology being considered in different contexts. Moreover, the literature gathered covers a wide range of topics, and it should be emphasized that the analytical methods and technologies utilized are typically consistent.

TABLE I. AN SUMMARY OF THE STUDIES UNDER CONSIDERATION FOR IORT APPLICATIONS.

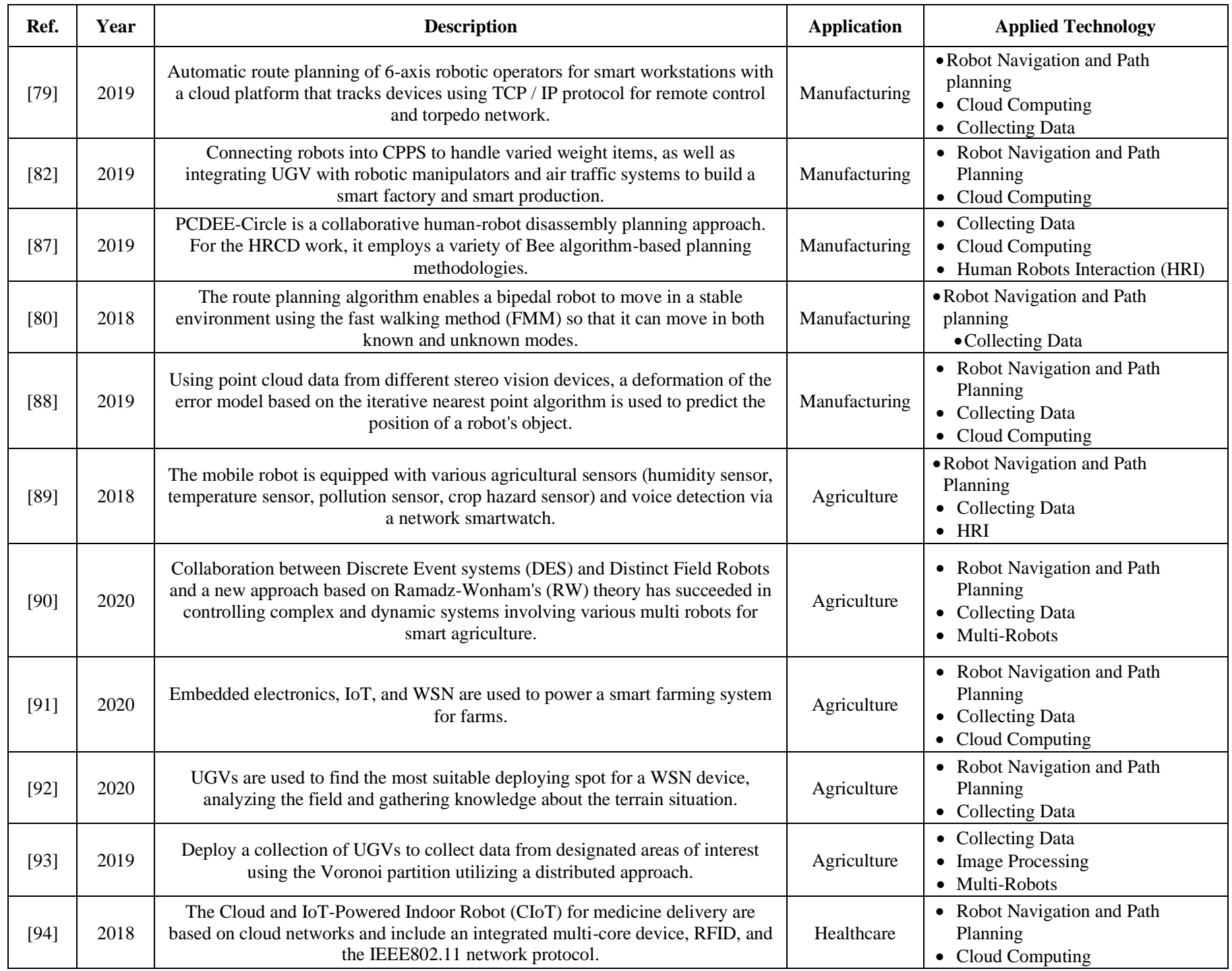




\begin{tabular}{|c|c|c|c|c|}
\hline [96] & 2018 & $\begin{array}{l}\text { Autonomous Networked Robots (ANR) for surveillance, RF transceivers for } \\
\text { networking and communication, with WSN implementation, and any sensor } \\
\text { node comprised of smoke, infrared fire, odor, and motion sensors. }\end{array}$ & Surveillance & $\begin{array}{l}\text { - Robot Navigation and Path } \\
\text { Planning } \\
\text { - Collecting Data } \\
\text { - Multi-Robots } \\
\end{array}$ \\
\hline [97] & 2018 & $\begin{array}{l}\text { Mines and hazardous gases were detected using a multipurpose field monitoring } \\
\text { robot. NodeMCU is an abbreviation for NodeMCU WiFi, which is used to link } \\
\text { controllers and build robots capable of navigating and collecting data on any } \\
\text { terrain. The data collected by the robot's sensors are transferred to cloud servers. }\end{array}$ & Surveillance & $\begin{array}{l}\text { - Collecting Data } \\
\text { - Cloud Computing }\end{array}$ \\
\hline [98] & 2018 & $\begin{array}{c}\text { InterBot } 1.0 \text { is an IoT-based robot system that is fitted for both long-range and } \\
\text { short-range communication devices. For smart surveillance, the robot is } \\
\text { effective at tracking real-time environments. }\end{array}$ & Surveillance & $\begin{array}{l}\text { - Robot Navigation and Path } \\
\text { Planning } \\
\text { - Collecting Data. }\end{array}$ \\
\hline
\end{tabular}

\section{A. Manufacture}

Robot-based processing is the foundation of smart manufacturing [75]. Manufacturing is adopting Industry 4.0 concepts such as sensor integration, robotics, and product and process control. The Fourth Industrial Revolution has changed the way goods are produced [76].

Smart manufacturing entails device versatility, management, and transition adaptation. Especially in terms of manufacturing processes, additive manufacturing is a crucial process. Indeed, breakthroughs in digital technology coming during the fourth industrial revolution must maintain pace with advancements in manufacturing processes and materials. In this environment, flexible additive electronics and their process robustness are important aspects for enabling smart production with sensor systems [77].

IoRT can involve many industrial uses, such as spot welding and spray painting [78]. Path mapping and navigation are critical for many manufacturing robots [79] [80]. It is important to increase the route planning algorithm's performance and to incorporate a cloud computing solution [81]. Using embedded CPSs, IoRT technologies will improve the robotic assembly process [82]. Industrial robots can overcome many knowledge and learning limitations by exchanging data with the cloud, uploading data about production architecture and other robots to the system [83].

The computing power of the cloud servers compensates for the reduced computing power of the robotic system. Cloud robotics usage and development have changed considerably, boosting the convenience and scalability of production job scheduling. The robotic assembly line has become a robotic assembly line where robots perform tasks that were previously performed by human labor. It has been made robotic [84].

Collaborative robots are stable, easy to use, and can support human operators, while the robot is in motion, the location of the person and the position of the hands should be calculated in real-time [85]. Methods based on inertial sensors and vision can be used to identify human activity in interactive work environments. The use of IoRT systems in smart manufacturing attempts to capabilities for individual limitations by utilizing the skills of robots and human operators [86].

\section{B. Agriculture}

IoRT systems may be built and deployed to provide farm technology that integrates resources, goods, and information [89]. Climate-sensitive agriculture (CSA) and sustainable intensification (SI) are the fundamental ideas of smart agriculture. CSA systems based on IoRT architecture may be used to develop the most resistant crop types to moisture, drought, floods, and salinity [99] [100]. SI exists to identify the optimum answer to resource scarcity and environmental protection by collecting and cultivating arable land under changing conditions, as well as to balance agricultural yields and environmental needs. The CSA's primary goal is to boost incomes and food stability by eliminating poverty.

SI aims to capitalize on crop complementarities by using the variety of crop types in the region. It also seeks to solve the problem of climate change adaptation, as farmers are unlikely to follow practices that would not result in higher yields in the short term [101][102].

Robotic systems in agriculture can be very useful for obtaining qualitative-quantitative material [103]. Integrating IoRT systems into agricultural machinery needs a novel method for managing feedback signals from the control system to the actuators [104]. IoRT-based smart farming systems are designed for various agricultural activities such as moisture-managing, irrigation, field control, and pest and animal protection. Such schemes can increase economic feasibility while reducing environmental effects and rising food security [105].

Precision seeding is the use of an IoRT strategy for herbicide, fertilization, or irrigation [93][90]. A WSN system consists of a small number of nodes containing radio frequency transceivers, microcontrollers, sensors, and power supplies [106]. IoT sensors are installed in a particular region to monitor environmental parameters via a wireless link and automatically sending data via multi-hop communication [13]. It is critical to install the node sensors in a heterogeneous manner to achieve the greatest coverage with the least amount of energy consumption to maximize yields and reduce costs [107]. 
WSN technologies are important in smart farming because they can self-regulate, diagnose, and organize themselves [108]. These characteristics allow great geographical and temporal resolution for crop monitoring in agricultural areas utilizing sensor nodes [109]. The hand robot autonomously configures the sensor network [94]. With this information, a robotic device can deliver water, fertilizer, and insecticides to more precise volumes and places [110].

\section{Health-Care}

Combining robots with IoT sensors and devices has several benefits in detecting patient issues and giving real-time health information, reducing the risk of human mistakes, such as medication, dosage, and treatment misdiagnosis [111]. Furthermore, IoRT technology has the potential to help a variety of different applications, including patient, staff, and ambulance monitoring, as well as automated data gathering and discovery [95][102].

\section{Education}

Robots in education must utilize intelligent and adaptable tactics to build and sustain rewarding social interactions with humans, as well as to provide support services such as homework and learning [112]. HRI may be assessed using electrodermal activity (EDA), which is a change in skin conductivity that occurs in response to concentration, anxiety, and arousal [113]. Furthermore, because children cannot respond to these impulses as adults do, the EDA response in children may deviate greatly from the average response in adults [114]. In such circumstances, IoRT systems used in children's education must gather, store, and evaluate data to create automatic predictions about the activities and states of the children [102].

\section{E. Surveillance}

Monitoring of sites and individuals is a well-known problem to regulate human wellbeing and maintain those ecosystems. IoRT systems are crucial in this context because they may give intelligent technologies for extensive monitoring in sensitive regions, prisons, military boundaries, public places, and houses [115][116]. Closed-circuit television CCTV cameras are commonly used to monitor the surroundings, both indoors and out [117]. However, such technology has several issues and limits, primarily because of the possibility of manipulation and the presence of blind areas. These drawbacks can be mitigated in part by increasing the number of cameras in the system which covers more corners at the expense of increasing the system's cost and complexity [118].

Enabling and deploying IoRT applications is frequently ideal in surveillance environments since IoRT systems may be created and deployed rapidly and reliably to cover vast regions to safeguard a particular region [98]. Additionally, cloud systems linked with robotics for real-time monitoring are especially effective for remote monitoring and human presence detection of environments such as homes, enterprises [97][119][120][121]. Measurements from sensors such as GPS, magnetic field, air quality, and environmental values also help in monitoring interior and outdoor conditions, since the robot may send data in real-time during a surveillance mission [122][96] [123][124][102].

\section{DISCUSSION}

As the first ICT revolution (from the desktop computer to the internet, to smartphones and wearable devices, to the Cloud and Internet of things) qualitatively enhanced data management, personal robot technologies would enable a similar drastic jump in their ability to function in the real world. The convergence of robotics with the IoT and Artificial Intelligence would be critical. IoT provides the ability to communicate with various stakeholders such as applications, smartphones, and people contact, providing the right option for multiple application domains. The combination of robotics, IoT, and artificial intelligence results in robots that can execute more complicated tasks independently or in collaboration with humans. In an IoT system, several robots can be conveniently integrated among themselves, as well as with objects and people, allowing data to be transferred to them without the need for human-to-computer or human-to-human contact. Basic theory derived from the application of artificial intelligence, including the use of cloud services [78], for instance, has a positive impact on device performance and dependability, and also user protection and responsive physical and behavioral human-robot interaction/collaboration.

Route planning is currently a challenge for industrial research, as the ability to locate and navigate independently is crucial for many industrial robots [79]. The assembly process is the process of integrating various components to create a new subcomponent or final product. IoRT technologies can improve such a process by utilizing integrated CPS, which intends to employ several robots to reposition or move components and execute more sophisticated assembly operations [82].

The main objective of the agricultural management approach is to efficiently reduce agricultural resources by limiting agricultural production costs and maximizing yield. To do this, a smart sensor network may be utilized to monitor and measure any changes in plants using a smart sensor network [91]. In [92], a sensor network is deployed automatically by a mobile robot, which is used to find the optimal location of the distribution node sensors to collect as much data from the environment as possible.

Especially IoRT is used in various applications, especially for people with mental disorders, paralysis, patients, disabilities, etc. used for patients. For example, it can provide medical, social, and economic benefits to groups of patients with special needs [94]. Additionally, IoRT technologies can offer many benefits, such as automated data collection and detection, as well as other applications such as patient, staff, and ambulance monitoring [95].

It is a known problem to monitor places and people to monitor human health and maintain certain environmental conditions. IoRT systems play an important role in this context since they may provide smart technologies for extensive monitoring in situations such as sensitive locations, hospitals, military boundaries, public places, and households [115]. Furthermore, cloud technologies combined with robots that provide real-time sensing are particularly beneficial for remote monitoring of surroundings such as homes, industrial facilities, retail stores, and wholesalers in a common scenario, among others [97]. 
Fig. 4 shows a graphical overview of the IoRT studies described. IoRT has a wide range of applications in the industrial and agricultural sectors (Fig. 4).

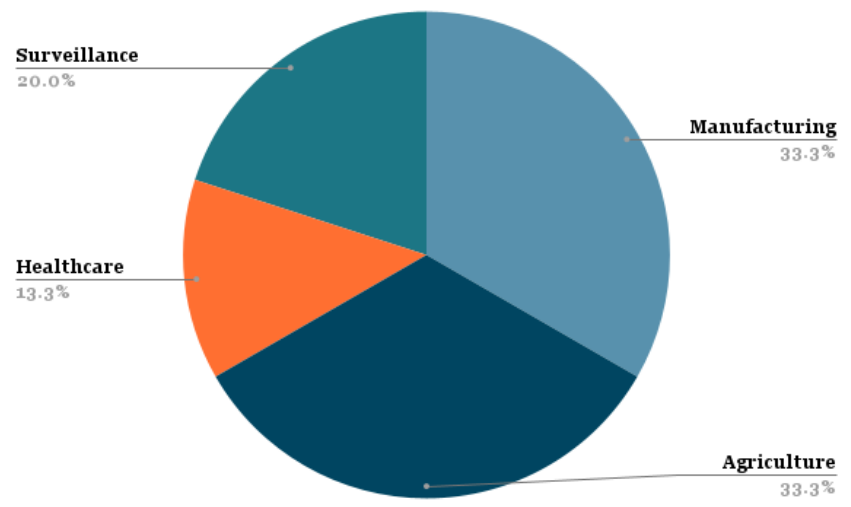

Fig. 4. A graphical illustration of the IoRT applications.

\section{VIII.CONCLUSION}

In this review, we highlight the latest research on IoT and IoRT by analyzing over 100 studies published over the years. IoRT systems are extremely useful in the sectors of health, education, and monitoring. Integrating robots with IoT sensors and devices gives several benefits, including real-time health data. To achieve and maintain adequate social interaction with humans, robots in education required the use of appropriate and adaptive actions. Surveillance of locations and people is a wellknown issue for monitoring human health and protecting particular environmental conditions. Surveillance of locations and people is a well-known issue for monitoring human health and protecting particular environmental conditions. IoRT system provides intelligent technologies for monitoring environments such as military areas, hospitals, houses, and public places. Sensor readings may be helpful for example air quality, environmental values, and GPS.

To present the concept of the Robotic Internet of Things, a synopsis of how CPS addresses the interaction between the actual and virtual worlds has been prepared. Many IoRT applications in various smart sectors have been detailed, demonstrating how typical robotic applications are separated from IoRT-based systems. Today, IoRT systems are required in a wide range of settings, from industrial sectors such as manufacturing and agriculture to daily life settings such as healthcare, education, and surveillance. It has been found that the IoRT method's design may be used to appropriately satisfy the demand for remote work.

This review focuses on IoRT-based systems that are utilized in production and development environments. The challenges of IoRT, in particular, have been thoroughly researched, demonstrating the growing need for heterogeneous robotic networks, human-robot interfaces for human-centered communication, energy management for productivity, and robot collaboration in cybersecurity to protect confidential information. Enhancements, reliability, and security are expected to provide new possibilities, especially new workplaces that will gain from the dispersion of modern robotics and networking technology.

\section{REFERENCES}

[1] P. P. Ray, "Internet of Robotic Things: Concept, Technologies, and Challenges," IEEE Access, vol. 4, pp. 9489-9500, 2016, doi: 10.1109/ACCESS.2017.2647747.

[2] A. Sanfeliu, N. Hagita, and A. Saffiotti, "Network robot systems," Robot. Auton. Syst., vol. 56, no. 10, pp. 793-797, 2008, doi: 10.1016/j.robot.2008.06.007.

[3] A. Saffiotti et al., The PEIS-Ecology project: Vision and results. 2008. doi: 10.1109/IROS.2008.4650962.

[4] J. Kim, K. Lee, Y. Kim, N. S. Kuppuswamy, and J. Jo, "Ubiquitous Robot: A New Paradigm for Integrated Services," in Proceedings 2007 IEEE International Conference on Robotics and Automation, 2007, pp. 28532858. doi: 10.1109/ROBOT.2007.363904.

[5] Y. G. Ha, J. C. Sohn, Y. J. Cho, and H. Yoon, "Towards a ubiquitous robotic companion: Design and implementation of ubiquitous robotic service framework," ETRI J., vol. 27, no. 6, pp. 666-676, 2005, doi: 10.4218/etrij.05.0105.0138.

[6] C. Homes and S. Meyer, "A Survey of Research on," pp. 1-10, 2010.

[7] K. Kamei, S. Nishio, N. Hagita, and M. Sato, "Cloud networked robotics," IEEE Netw. - Netw., vol. 26, pp. 28-34, May 2012, doi: 10.1109/MNET.2012.6201213.

[8] P. Simoens, M. Dragone, and A. Saffiotti, "The Internet of Robotic Things: A review of concept, added value and applications," Int. J. Adv. Robot. Syst., vol. 15, no. 1, pp. 1-11, 2018, doi: 10.1177/1729881418759424.

[9] A. Roy Chowdhury, "IoT and Robotics: a synergy," PeerJ Prepr., vol. 5, p. e2760v1, 2017, doi: 10.7287/peerj.preprints.2760v1.

[10] C. Razafimandimby, V. Loscri, and A. M. Vegni, “A Neural Network and IoT Based Scheme for Performance Assessment in Internet of Robotic Things," in 2016 IEEE First International Conference on Internet-ofThings Design and Implementation (IoTDI), 2016, pp. 241-246. doi: 10.1109/IoTDI.2015.10.

[11] P. Bhavanam and Mrs. T. Jami, "PERFORMANCE ASSESSMENT IN INTERNET OF ROBOTIC THINGS BASED ON IOT," Int. J. Innov. Technol. Res. Vol 5 No 32017 April - May 2017, Jun. 2017.

[12] J. Oh, Y. Park, J. Choi, and J. Choi, "A rule-based context transforming model for robot services in internet of things environment," in 2017 14th International Conference on Ubiquitous Robots and Ambient Intelligence (URAI), 2017, pp. 331-336. doi: 10.1109/URAI.2017.7992744.

[13] V. Scilimati et al., "Industrial Internet of things at work: a case study analysis in robotic-aided environmental monitoring," IET Wirel. Sens. Syst., vol. 7, no. 5, pp. 155-162, Oct. 2017, doi: https://doi.org/10.1049/ietwss.2017.0032.

[14] L. Atzori, A. Iera, and G. Morabito, "The Internet of Things: A survey," Comput. Netw., vol. 54, no. 15, pp. 2787-2805, 2010, doi: https://doi.org/10.1016/j.comnet.2010.05.010.

[15] K. Jacksi and S. Badiozamany, "General method for data indexing using clustering methods," Int J Sci Eng, vol. 6, no. 3, pp. 641-644, 2015.

[16] N. M. Salih and K. Jacksi, "State of the art document clustering algorithms based on semantic similarity," J. Inform., vol. 14, no. 2, pp. 58-75, 2020.

[17] W. Shi, J. Cao, Q. Zhang, Y. Li, and L. Xu, "Edge Computing: Vision and Challenges," IEEE Internet Things J., vol. 3, no. 5, pp. 637-646, 2016, doi: 10.1109/JIOT.2016.2579198.

[18] B. Kehoe, S. Patil, P. Abbeel, and K. Goldberg, "A Survey of Research on Cloud Robotics and Automation," IEEE Trans. Autom. Sci. Eng., vol. 12, no. 2, pp. 398-409, 2015, doi: 10.1109/TASE.2014.2376492.

[19] G. Mohanarajah, D. Hunziker, R. D'Andrea, and M. Waibel, "Rapyuta: A Cloud Robotics Platform," IEEE Trans. Autom. Sci. Eng., vol. 12, no. 2, pp. 481-493, 2015, doi: 10.1109/TASE.2014.2329556.

[20] T. Cieslewski, S. Lynen, M. Dymczyk, S. Magnenat, and R. Siegwart, "Map API - scalable decentralized map building for robots," in 2015 IEEE International Conference on Robotics and Automation (ICRA), 2015, pp. 6241-6247. doi: 10.1109/ICRA.2015.7140075.

[21] M. Szlenk, C. Zieliński, M. Figat, and T. Kornuta, "Reconfigurable agent architecture for robots utilising cloud computing," Adv. Intell. Syst. 
Comput., vol. 351, pp. 253-264, 2015, doi: 10.1007/978-3-319-15847$1 \_25$.

[22] R. S. Batth, A. Nayyar, and A. Nagpal, "Internet of Robotic Things: Driving Intelligent Robotics of Future - Concept, Architecture, Applications and Technologies," Proc. - 4th Int. Conf. Comput. Sci. ICCS 2018, no. August, pp. 151-160, 2019, doi: 10.1109/ICCS.2018.00033.

[23] L. Reinfurt, U. Breitenbücher, M. Falkenthal, F. Leymann, and A. Riegg, "Internet of things patterns," ACM Int. Conf. Proceeding Ser., 2016, doi: 10.1145/3011784.3011789.

[24] S. Qanbari et al., "IoT design patterns: Computational constructs to design, build and engineer edge applications," Proc. - 2016 IEEE 1st Int. Conf. Internet--Things Des. Implement. IoTDI 2016, pp. 277-282, 2016, doi: 10.1109/IoTDI.2015.18.

[25] H. Washizaki et al., "Landscape of IoT Patterns," in 2019 IEEE/ACM 1st International Workshop on Software Engineering Research \& Practices for the Internet of Things (SERP4IoT), 2019, pp. 57-60. doi: 10.1109/SERP4IoT.2019.00017.

[26] G. Bloom, B. Alsulami, E. Nwafor, and I. C. Bertolotti, "Design patterns for the industrial Internet of Things," IEEE Int. Workshop Fact. Commun. Syst. - Proc. WFCS, vol. 2018-June, pp. 1-10, 2018, doi: 10.1109/WFCS.2018.8402353.

[27] A. Ghosh, D. J. Edwards, and M. R. Hosseini, "Patterns and trends in Internet of Things (IoT) research: future applications in the construction industry," Eng. Constr. Archit. Manag., vol. 28, no. 2, pp. 457-481, 2020, doi: 10.1108/ECAM-04-2020-0271.

[28] G. S. VIRK, H. S. PARK, S. YANG, and J. WANG, "ISO MODULARITY FOR SERVICE ROBOTS $<$ xref ref-type="fn" rid="B979813149137_0077FN001" ptype="B" citart="citart1" $>$ *</xref $>$," in Advances in Cooperative Robotics, WORLD SCIENTIFIC, 2016, pp. 663-671. doi: doi:10.1142/9789813149137_0077.

[29] R. Damaševičius, G. Majauskas, and V. Štuikys, "Application of design patterns for hardware design," Proc. - Des. Autom. Conf., no. 370 37, pp. 48-53, 2003, doi: 10.1145/775832.775847.

[30] V. Malcher, "Design Patterns in Cloud Computing," in 2015 10th International Conference on P2P, Parallel, Grid, Cloud and Internet Computing (3PGCIC), 2015, pp. 32-35. doi: 10.1109/3PGCIC.2015.142.

[31] M. Bartoletti and L. Pompianu, "An Empirical analysis of smart contracts: Platforms, applications, and design patterns," Lect. Notes Comput. Sci. Subser. Lect. Notes Artif. Intell. Lect. Notes Bioinforma., vol. 10323 LNCS, pp. 494-509, 2017, doi: 10.1007/978-3-319-70278-0_31.

[32] L. Reinfurt, U. Breitenbücher, M. Falkenthal, F. Leymann, and A. Riegg, "Internet of Things Patterns for Devices: Powering, Operating, and Sensing," Int. J. Adv. Internet Technol., pp. 106-123, 2017.

[33] L. Reinfurt, U. Breitenbücher, M. Falkenthal, F. Leymann, and A. Riegg, Internet of things patterns for communication and management, vol. 10600 LNCS. 2019. doi: 10.1007/978-3-030-14291-9_5.

[34] G. Meszaros and J. Doble, "MetaPatterns: A Pattern Language for Pattern Writing," Pattern Lang. Program Des., pp. 1-36, 1997.

[35] C. Fehling et al., "Institute of Architecture of Application Systems Authoring, and Application A Process for Pattern Identification , Authoring, and Application," 2015.

[36] M. Mazzara, I. Afanasyev, S. R. Sarangi, S. Distefano, V. Kumar, and M. Ahmad, "A Reference Architecture for Smart and Software-Defined Buildings," in 2019 IEEE International Conference on Smart Computing (SMARTCOMP), 2019, pp. 167-172. doi: 10.1109/SMARTCOMP.2019.00048.

[37] I. Afanasyev et al., "Towards the internet of robotic things: Analysis, architecture, components and challenges," Proc. - Int. Conf. Dev. ESystems Eng. DeSE, vol. October-20, pp. 3-8, 2019, doi: 10.1109/DeSE.2019.00011.

[38] O. Vermesan et al., "Internet of robotic things: converging sensing/actuating, hypoconnectivity, artificial intelligence and IoT Platforms," in Cognitive Hyperconnected Digital Transformation: Internet of Things Intelligence Evolution, J. B. Ovidiu Vermesan, Ed. School of Science and Technology, Örebro University: River Publishers, 2017, pp. 97-155.

[39] E. Menegatti, Advances in Intelligent Systems and Computing 302 Intelligent Autonomous Systems 13.
[40] I. S. of R. and A. T. C. On and N. Robots., "Networked Robots." https://www.ieee-ras.org/technical-committees/117-technicalcommittees/networked-robots/146-networked-robots

[41] M. J. Mataric, “EMBEDDING ROBOTS,” vol. 43, pp. 67-74.

[42] G. Hu, W. P. Tay, and Y. Wen, "Cloud robotics: architecture, challenges and applications," IEEE Netw., vol. 26, no. 3, pp. 21-28, 2012, doi: 10.1109/MNET.2012.6201212.

[43] M. Waibel et al., "Robo earth," IEEE Robot. Autom. Mag., vol. 18, no. 2, pp. 69-82, 2011, doi: 10.1109/MRA.2011.941632.

[44] Vermesan and Ovidiu, Digitising the Industry Internet of Things Connecting the Physical, Digital and Virtual Worlds. 2016.

[45] F. Cavallo et al., "Development of a Socially Believable Multi-Robot Solution from Town to Home," Cogn. Comput., vol. 6, no. 4, pp. 954-967, 2014, doi: 10.1007/s12559-014-9290-z.

[46] B. Mutlu and J. Forlizzi, "Robots in organizations: The role of workflow, social, and environmental factors in human-robot interaction," in $20083 \mathrm{rd}$ ACM/IEEE International Conference on Human-Robot Interaction (HRI), 2008, pp. 287-294. doi: 10.1145/1349822.1349860.

[47] F. Yazdani, B. Brieber, M. Beetz, and F. Yazdani, "Cognition-enabled Robot Control for Mixed Human-Robot Rescue Teams Simulation-based Rescue Scenario Cognition-enabled Robot Control in a Human-Robot Team 2 Artificial Intelligence," no. July, 2014.

[48] X. J. Liu, "Research toward IoT and Robotics in Intelligent Manufacturing: A Survey," Int. J. Mater. Mech. Manuf., vol. 7, no. 3, pp. 128-132, 2019, doi: 10.18178/ijmmm.2019.7.3.445.

[49] L. Hu, Y. Miao, G. Wu, M. M. Hassan, and I. Humar, "iRobot-Factory: An intelligent robot factory based on cognitive manufacturing and edge computing," Future Gener. Comput. Syst., vol. 90, pp. 569-577, 2019, doi: https://doi.org/10.1016/j.future.2018.08.006.

[50] J. Reis, "Towards an Industrial Agent Oriented Approach," Dr. Symp. Inform. Eng., no. January, pp. 1-12, 2014.

[51] V. Rampa, F. Vicentini, S. Savazzi, N. Pedrocchi, M. Ioppolo, and M. Giussani, "Safe human-robot cooperation through sensor-less radio localization," in 2014 12th IEEE International Conference on Industrial Informatics (INDIN), 2014, pp. 683-689. doi: 10.1109/INDIN.2014.6945596.

[52] K. Qian, X. Ma, X. Dai, F. Fang, and B. Zhou, "Decision-Theoretical Navigation of Service Robots Using POMDPs with Human-Robot CoOccurrence Prediction," Int. J. Adv. Robot. Syst., vol. 10, no. 2, p. 143, Feb. 2013, doi: 10.5772/55926.

[53] Z. N. Rashid, S. R. Zebari, K. H. Sharif, and K. Jacksi, "Distributed cloud computing and distributed parallel computing: A review," 2018, pp. 167172.

[54] I. M. Ibrahim et al., "Task scheduling algorithms in cloud computing: A review," Turk. J. Comput. Math. Educ., vol. 12, no. 4, pp. 1041-1053, 2021.

[55] B. Siciliano and O. Khatib, Robotics and the handbook. 2016. doi: 10.1007/978-3-319-32552-1_1.

[56] R. C. Luo, C.-C. Yih, and K. L. Su, "Multisensor fusion and integration: approaches, applications, and future research directions," IEEE Sens. J., vol. 2, no. 2, pp. 107-119, 2002, doi: 10.1109/JSEN.2002.1000251.

[57] S. M. LaValle, "Planning algorithms," Plan. Algorithms, vol. 9780521862 , pp. 1-826, 2006, doi: 10.1017/CBO9780511546877.

[58] X. Lv, M. Zhang, and H. Li, "Robot control based on voice command," in 2008 IEEE International Conference on Automation and Logistics, 2008, pp. 2490-2494. doi: 10.1109/ICAL.2008.4636587.

[59] A. Mosavi and A. Varkonyi-Koczy, "Learning in Robotics," Int. J. Comput. Appl., vol. 157, pp. 975-8887, Jan. 2017, doi: 10.5120/ijca2017911661.

[60] C. Razafimandimby et al., "Towards efficient deployment in Internet of Robotic Things To cite this version: HAL Id: hal-01499345 Towards efficient deployment in," 2017.

[61] R. R. Zebari, S. R. M. Zeebaree, A. B. Sallow, H. M. Shukur, O. M. Ahmad, and K. Jacksi, "Distributed Denial of Service Attack Mitigation using High Availability Proxy and Network Load Balancing," in 2020 International Conference on Advanced Science and Engineering 
(ICOASE), Dec. 2020, pp. 174-179. doi: 10.1109/ICOASE51841.2020.9436545.

[62] R. R. Zebari, S. R. Zeebaree, and K. Jacksi, "Impact Analysis of HTTP and SYN Flood DDoS Attacks on Apache 2 and IIS 10.0 Web Servers," 2018, pp. $156-161$.

[63] V. Matellán, T. Bonaci, and G. Sabaliauskaite, "Cyber-security in robotics and autonomous systems," Robot. Auton. Syst., vol. 100, pp. 41-42, 2018, doi: https://doi.org/10.1016/j.robot.2017.10.020.

[64] M. A. Sadeeq, S. R. Zeebaree, R. Qashi, S. H. Ahmed, and K. Jacksi, "Internet of Things security: a survey," 2018, pp. 162-166.

[65] FRANK L. LEWIS, Autonomous Mobile Robots, vol. 53, no. 9. 2013.

[66] O. Palinko, F. Rea, G. Sandini, and A. Sciutti, "Robot reading human gaze: Why eye tracking is better than head tracking for human-robot collaboration," in 2016 IEEE/RSJ International Conference on Intelligent Robots and Systems (IROS), 2016, pp. 5048-5054. doi: 10.1109/IROS.2016.7759741.

[67] P. Hunter, "Remote working in research," EMBO Rep., vol. 20, no. 1, p. e47435, Jan. 2019, doi: https://doi.org/10.15252/embr.201847435.

[68] M. J. Islam and J. Sattar, "Mixed-domain biological motion tracking for underwater human-robot interaction," in 2017 IEEE International Conference on Robotics and Automation (ICRA), 2017, pp. 4457-4464. doi: 10.1109/ICRA.2017.7989516.

[69] R. PATEL, "Human Robot Interaction With Cloud Assisted Voice Control," no. May, 2018.

[70] G. d. Campo, S. Calatrava, G. Cañada, J. Olloqui, R. Martinez, and A. Santamaria, "IoT Solution for Energy Optimization in Industry 4.0: Issues of a Real-life Implementation," in 2018 Global Internet of Things Summit (GIoTS), 2018, pp. 1-6. doi: 10.1109/GIOTS.2018.8534537.

[71] L. Bukata, P. Šủcha, Z. Hanzálek, and P. Burget, "Energy Optimization of Robotic Cells," IEEE Trans. Ind. Inform., vol. 13, no. 1, pp. 92-102, 2017, doi: 10.1109/TII.2016.2626472.

[72] N. Dragoni, A. Giaretta, and M. Mazzara, "The internet of hackable things," Adv. Intell. Syst. Comput., vol. 717, pp. 129-140, 2018, doi: 10.1007/978-3-319-70578-1_13.

[73] A. Khalid, P. Kirisci, Z. H. Khan, Z. Ghrairi, K.-D. Thoben, and J. Pannek, "Security framework for industrial collaborative robotic cyber-physical systems," Comput. Ind., vol. 97, pp. 132-145, 2018, doi: https://doi.org/10.1016/j.compind.2018.02.009.

[74] A. Wegner, J. Graham, and E. Ribble, "A New Approach to Cyberphysical Security in Industry 4.0," pp. 59-72, 2017, doi: 10.1007/978-3-319-506609_3.

[75] M. R. Pedersen et al., "Robot skills for manufacturing: From concept to industrial deployment," Robot. Comput.-Integr. Manuf., vol. 37, pp. 282291, 2016, doi: https://doi.org/10.1016/j.rcim.2015.04.002.

[76] P. D. Urbina Coronado, H. Ahuett-Garza, R. Morales-Menendez, P. Orta Castañón, L. D. Dávila, and M. R. Flores Escalera, "Connectivity of a modular electric vehicle by the use of a mobile device," Adv. Mech. Eng., vol. 9, no. 7, p. 1687814017708087, Jul. 2017, doi: $10.1177 / 1687814017708087$.

[77] A. Kumar, "Methods and Materials for Smart Manufacturing: Additive Manufacturing, Internet of Things, Flexible Sensors and Soft Robotics," Manuf. Lett., vol. 15, pp. 122-125, 2018, doi: https://doi.org/10.1016/j.mfglet.2017.12.014.

[78] A. Grau, M. Indri, L. L. Bello, and T. Sauter, "Industrial robotics in factory automation: From the early stage to the Internet of Things," in IECON 2017 - 43rd Annual Conference of the IEEE Industrial Electronics Society, 2017, pp. 6159-6164. doi: 10.1109/IECON.2017.8217070.

[79] W. Chang, S. Lin, J. Hsu, and B. Hsu, "Automatic Path Planning of Robot for Intelligent Manufacturing Based on Network Remoted Controlling and Simulation," in 2019 4th Asia-Pacific Conference on Intelligent Robot Systems (ACIRS), 2019, pp. 164-168. doi: 10.1109/ACIRS.2019.8935967.

[80] K. Mrudul, R. K. Mandava, and P. R. Vundavilli, "An Efficient Path Planning Algorithm for Biped Robot using Fast Marching Method," Procedia Comput. Sci., vol. 133, pp. 116-123, 2018, doi: https://doi.org/10.1016/j.procs.2018.07.015.

[81] L. Xin, H. Xiangyuan, Y. Ziqi, Q. Xiaoning, and D. Yingkui, "The Algebraic Algorithm for Path Planning Problem of AGV in Flexible
Manufacturing System," in 2018 37th Chinese Control Conference (CCC), 2018, pp. 2396-2399. doi: 10.23919/ChiCC.2018.8483105.

[82] Q. Liu, P. Hua, A. Sultan, L. Shen, E. Mueller, and F. Boerner, "Study of the Integration of Robot in Cyber-Physical Production Systems," in 2019 International Conference on Cyber-Enabled Distributed Computing and Knowledge Discovery (CyberC), 2019, pp. 367-370. doi: 10.1109/CyberC.2019.00069.

[83] X. V. Wang, L. Wang, A. Mohammed, and M. Givehchi, "Ubiquitous manufacturing system based on Cloud: A robotics application," Robot. Comput.-Integr. Manuf., vol. 45, pp. 116-125, 2017, doi: https://doi.org/10.1016/j.rcim.2016.01.007.

[84] J. Wan, S. Tang, H. Yan, D. Li, S. Wang, and A. V Vasilakos, "Cloud robotics: Current status and open issues," IEEE Access, vol. 4, pp. 2797 2807, 2016, doi: 10.1109/ACCESS.2016.2574979.

[85] R.-J. Halme, M. Lanz, J. Kämäräinen, R. Pieters, J. Latokartano, and A. Hietanen, "Review of vision-based safety systems for human-robot collaboration," Procedia CIRP, vol. 72, pp. 111-116, 2018, doi: https://doi.org/10.1016/j.procir.2018.03.043.

[86] M. A. R. Garcia, R. Rojas, L. Gualtieri, E. Rauch, and D. Matt, "A humanin-the-loop cyber-physical system for collaborative assembly in smart manufacturing," Procedia CIRP, vol. 81, pp. 600-605, 2019, doi: https://doi.org/10.1016/j.procir.2019.03.162.

[87] Q. Liu, Z. Liu, W. Xu, Q. Tang, Z. Zhou, and D. T. Pham, "Human-robot collaboration in disassembly for sustainable manufacturing," Int. J. Prod. Res., vol. 57, no. 12, pp. 4027-4044, Jun. 2019, doi: 10.1080/00207543.2019.1578906.

[88] F. Chen, M. Selvaggio, and D. G. Caldwell, "Dexterous Grasping by Manipulability Selection for Mobile Manipulator With Visual Guidance," IEEE Trans. Ind. Inform., vol. 15, no. 2, pp. 1202-1210, 2019, doi: 10.1109/TII.2018.2879426.

[89] D.Saravanan, G. L. Archana, and R.Parthiban, "VOICE CONTROLLED HUMANOID ROBOTIC CAR FOR SMART AGRICULTURE USING ARDUINO AND ANDROID SMART WATCH," vol. 119, no. 14, pp. 829-833, 2018.

[90] C. Ju and H. I. Son, "Modeling and Control of Heterogeneous Agricultural Field Robots Based on Ramadge-Wonham Theory," IEEE Robot. Autom. Lett., vol. 5, no. 1, pp. 48-55, 2020, doi: 10.1109/LRA.2019.2941178.

[91] M. Mahbub, "A smart farming concept based on smart embedded electronics, internet of things and wireless sensor network," Internet Things, vol. 9, p. 100161, 2020, doi: https://doi.org/10.1016/j.iot.2020.100161.

[92] L. Romeo et al., "Automated Deployment of IoT Networks in Outdoor Scenarios using an Unmanned Ground Vehicle," in 2020 IEEE International Conference on Industrial Technology (ICIT), 2020, pp. 369374. doi: 10.1109/ICIT45562.2020.9067099.

[93] S. Faryadi, M. Davoodi, and J. Mohammadpour Velni, "Agricultural Field Coverage Using Cooperating Unmanned Ground Vehicles." Oct. 08, 2019. doi: 10.1115/DSCC2019-8992.

[94] H. Cao, X. Huang, J. Zhuang, J. Xu, and Z. Shao, "CIoT-Robot Cloud and IoT Assisted Indoor Robot for Medicine Delivery BT - Proceedings of the 2018 Joint International Advanced Engineering and Technology Research Conference (JIAET 2018)," Mar. 2018, pp. 85-89. doi: https://doi.org/10.2991/jiaet-18.2018.14.

[95] H. Zhou, G. Yang, H. Lv, X. Huang, H. Yang, and Z. Pang, "IoT-Enabled Dual-Arm Motion Capture and Mapping for Telerobotics in Home Care," IEEE J. Biomed. Health Inform., vol. 24, no. 6, pp. 1541-1549, 2020, doi: 10.1109/JBHI.2019.2953885.

[96] C. Chukwuemeka and M. Habib, "Development of Autonomous Networked Robots (ANR) for Surveillance: Conceptual Design and Requirements," in IECON 2018 - 44th Annual Conference of the IEEE Industrial Electronics Society, 2018, pp. 3757-3763. doi: 10.1109/IECON.2018.8591452.

[97] M. Ashokkumar and T. Thirumurugan, "Integrated IOT based design and Android operated Multi-purpose Field Surveillance Robot for Military Use BT - Proceedings of the International Conference for Phoenixes on Emerging Current Trends in Engineering and Management (PECTEAM 2018)," Feb. 2018, pp. 236-243. doi: https://doi.org/10.2991/pecteam18.2018.42. 
[98] W. H. Mohd Saad, S. A. Abd. Karim, N. Azhar, Z. Manap, Y. Y. Soon, and M. Mat Ibrahim, "Line Follower Mobile Robot for Surveillance Camera Monitoring System,” J. Telecommun. Electron. Comput. Eng. JTEC, vol. 10, no. 2-7 SE-Articles, pp. 1-5, Jul. 2018.

[99] W. J. Blaser, J. Oppong, S. P. Hart, J. Landolt, E. Yeboah, and J. Six, "Climate-smart sustainable agriculture in low-to-intermediate shade agroforests," Nat. Sustain., vol. 1, no. 5, pp. 234-239, 2018, doi: $10.1038 / \mathrm{s} 41893-018-0062-8$

[100]B. M. Campbell, P. Thornton, R. Zougmoré, P. van Asten, and L. Lipper, "Sustainable intensification: What is its role in climate smart agriculture?," Curr. Opin. Environ. Sustain., vol. 8, pp. 39-43, 2014, doi: https://doi.org/10.1016/j.cosust.2014.07.002.

[101]I. Mat, M. R. M. Kassim, A. N. Harun, and I. M. Yusoff, "Smart Agriculture Using Internet of Things," in 2018 IEEE Conference on Open Systems (ICOS), 2018, pp. 54-59. doi: 10.1109/ICOS.2018.8632817.

[102] L. Romeo, A. Petitti, R. Marani, and A. Milella, "Internet of robotic things in smart domains: Applications and challenges," Sens. Switz., vol. 20, no. 12, pp. 1-23, 2020, doi: 10.3390/s20123355.

[103]V. Serebrenny, M. Shereuzhev, and I. Metasov, "Approaches to the robotization of agricultural mobile machines," MATEC Web Conf, vol. $161,2018$.

[104]T. Duckett et al., "Agricultural Robotics: The Future of Robotic Agriculture," 2018.

[105]M. S. Mekala and P. Viswanathan, "A Survey: Smart agriculture IoT with cloud computing," in 2017 International conference on Microelectronic Devices, Circuits and Systems (ICMDCS), 2017, pp. 1-7. doi: 10.1109/ICMDCS.2017.8211551

[106] G. Valecce et al., "Robotic-aided IoT: automated deployment of a 6TiSCH network using an UGV," IET Wirel. Sens. Syst., vol. 9, no. 6, pp. 438-446, Dec. 2019, doi: https://doi.org/10.1049/iet-wss.2019.0076.

[107]F. Adamo et al., "Comparison of current sensors for power consumption assessment of wireless sensors network nodes," in 2017 IEEE International Workshop on Measurement and Networking (M\&N), 2017, pp. 1-4. doi: 10.1109/IWMN.2017.8078390.

[108]G. Micoli et al., "ASAP: A Decentralized Slot Reservation Policy for Dynamic 6TiSCH Networks in Industrial IoT," in 2019 IEEE International Conference on Communications Workshops (ICC Workshops), 2019, pp. 1-6. doi: 10.1109/ICCW.2019.8757078.

[109] K. L. Krishna, O. Silver, W. F. Malende, and K. Anuradha, "Internet of Things application for implementation of smart agriculture system," in 2017 International Conference on I-SMAC (IoT in Social, Mobile, Analytics and Cloud) (I-SMAC), 2017, pp. 54-59. doi: 10.1109/ISMAC.2017.8058236.

[110]M. Roopaei, P. Rad, and K. R. Choo, "Cloud of Things in Smart Agriculture: Intelligent Irrigation Monitoring by Thermal Imaging," IEEE Cloud Comput., vol. 4, no. 1, pp. 10-15, 2017, doi: 10.1109/MCC.2017.5.

[111]R. Amin, S. K. H. Islam, G. P. Biswas, M. K. Khan, and N. Kumar, "A robust and anonymous patient monitoring system using wireless medical sensor networks," Future Gener. Comput. Syst., vol. 80, pp. 483-495, 2018, doi: https://doi.org/10.1016/j.future.2016.05.032.
[112]M. Chen, J. Zhou, G. Tao, J. Yang, and L. Hu, "Wearable Affective Robot," IEEE Access, vol. 6, pp. 64766-64776, 2018, doi: 10.1109/ACCESS.2018.2877919.

[113] T. Bonaci, J. Herron, T. Yusuf, J. Yan, T. Kohno, and H. J. Chizeck, “To Make a Robot Secure: An Experimental Analysis of Cyber Security Threats Against Teleoperated Surgical Robots," pp. 1-11, 2015.

[114]R. M. Fenning, J. K. Baker, B. R. Baucom, S. A. Erath, M. A. Howland, and J. Moffitt, "Electrodermal Variability and Symptom Severity in Children with Autism Spectrum Disorder," J. Autism Dev. Disord., vol. 47, no. 4, pp. 1062-1072, 2017, doi: 10.1007/s10803-016-3021-0.

[115] A. Nayyar, V. Puri, N. G. Nguyen, and D. N. Le, Smart surveillance robot for real-time monitoring and control system in environment and industrial applications, vol. 672. Springer Singapore, 2018. doi: 10.1007/978-98110-7512-4_23.

[116]R. Singh, R. Samkaria, A. Gehlot, and S. Choudhary, "Design and Development of IoT enabled Multi Robot System for Search and Rescue Mission," Int. J. Web Appl., vol. 10, no. 2, p. 51, 2018, doi: 10.6025/ijwa/2018/10/2/51-63.

[117] S. Saponara, L. Pilato, and L. Fanucci, "Exploiting CCTV camera system for advanced passenger services on-board trains," in 2016 IEEE International Smart Cities Conference (ISC2), 2016, pp. 1-6. doi: 10.1109/ISC2.2016.7580748.

[118] A. Petitti et al., "A Network of Stationary Sensors and Mobile Robots for Distributed Ambient Intelligence," IEEE Intell. Syst., vol. 31, no. 6, pp. 28 34, 2016, doi: 10.1109/MIS.2016.43.

[119] A. Khakimov, A. Muthanna, R. Kirichek, A. Koucheryavy, and M. S. A. Muthanna, "Investigation of methods for remote control IoT-devices based on cloud platforms and different interaction protocols," in 2017 IEEE Conference of Russian Young Researchers in Electrical and Electronic Engineering (EIConRus), 2017, pp. 160-163. doi: 10.1109/EIConRus.2017.7910518.

[120]H. S. Raju and S. Shenoy, "Real-time remote monitoring and operation of industrial devices using IoT and cloud," in 2016 2nd International Conference on Contemporary Computing and Informatics (IC3I), 2016, pp. 324-329. doi: 10.1109/IC3I.2016.7917983.

[121]S. Meghana, T. V Nikhil, R. Murali, S. Sanjana, R. Vidhya, and K. J. Mohammed, "Design and implementation of surveillance robot for outdoor security," in 2017 2nd IEEE International Conference on Recent Trends in Electronics, Information \& Communication Technology (RTEICT), 2017, pp. 1679-1682. doi: 10.1109/RTEICT.2017.8256885.

[122] K. Rajkumar, C. S. Kumar, C. Yuvashree, and S. Murugan, "PORTABLE SURVEILLANCE ROBOT USING IOT,” pp. 94-97, 2019.

[123] M. S. Shah and P. B. Borole, "Surveillance and rescue robot using Android smartphone and the Internet," in 2016 International Conference on Communication and Signal Processing (ICCSP), 2016, pp. 1526-1530. doi: 10.1109/ICCSP.2016.7754413.

[124]V. Wanjari and C. Kamargaonkar, "A Review Paper on IoT based Cognitive Robot for Military Surveillance,” pp. 2276-2278, 2019 